\title{
BMJ Open Modelling study to estimate the health burden of foodborne diseases: cases, general practice consultations and hospitalisations in the UK, 2009
}

\author{
Sarah J O'Brien, ${ }^{1,2}$ Tricia L Larose, ${ }^{3,4}$ Goutam K Adak, ${ }^{5}$ Meirion R Evans, ${ }^{6}$ \\ Clarence C Tam, ${ }^{3,7}$ On behalf of the Foodborne Disease Attribution Study Group
}

To cite: O'Brien SJ, Larose TL, Adak GK, et al. Modelling study to estimate the health burden of foodborne diseases: cases, general practice consultations and hospitalisations in the UK, 2009. BMJ Open 2016;6: e011119. doi:10.1136/ bmjopen-2016-011119

- Prepublication history and additional material is available. To view please visit the journal (http://dx.doi.org/ 10.1136/bmjopen-2016011119).

Received 11 January 2016 Revised 21 June 2016 Accepted 5 August 2016

CrossMark

For numbered affiliations see end of article.

Correspondence to Professor Sarah J 0’Brien; s.j.obrien@liverpool.ac.uk

\section{ABSTRACT}

Objective: To generate estimates of the burden of UK-acquired foodborne disease accounting for uncertainty.

Design: A modelling study combining data from national public health surveillance systems for laboratory-confirmed infectious intestinal disease (IID) and outbreaks of foodborne disease and 2 prospective, population-based studies of IID in the community. The underlying data sets covered the time period 1993-

2008. We used Monte Carlo simulation and a Bayesian approach, using a systematic review to generate Bayesian priors. We calculated point estimates with $95 \%$ credible intervals ( $\mathrm{Crl})$.

Setting: UK, 2009.

Outcome measures: Pathogen-specific estimates of the number of cases, general practice (GP) consultations and hospitalisations for foodborne disease in the UK in 2009.

Results: Bayesian approaches gave slightly more conservative estimates of overall health burden ( 511000 cases vs 566000 cases). Campylobacter is the most common foodborne pathogen, causing 280400 (95\% Crl 182 503-435 693) food-related cases and 38860 (95\% Crl 27 160-55 610) GP consultations annually. Despite this, there are only around 562 (95\% Crl 189-1330) food-related hospital admissions due to Campylobacter, reflecting relatively low disease severity. Salmonella causes the largest number of hospitalisations, an estimated 2490 admissions (95\% Crl 607-9631), closely followed by Escherichia coli 0157 with 2233 admissions (95\% Crl 170-32 159). Other common causes of foodborne disease include Clostridium perfringens, with an estimated 79570 cases annually $(95 \% \mathrm{Crl}$ 30 700-211 298) and norovirus with 74100 cases (95\% Crl 61 150-89 660). Other viruses and protozoa ranked much lower as causes of foodborne disease.

Conclusions: The 3 models yielded similar estimates of the burden of foodborne illness in the UK and show that continued reductions in Campylobacter,

Salmonella, E. coli 0157, C. perfringens and norovirus are needed to mitigate the impact of foodborne disease.

\section{Strengths and limitations of this study}

- This is the first burden of foodborne illness modelling study to incorporate empirical data and prior information from a systematic review together with Bayesian methodology for estimating the proportion of infectious intestinal disease that is transmitted through contaminated food.

- Our estimates are based on high-quality data sets, including directly observed, pathogenspecific incidence data.

- Our methods take full account of parameter uncertainties.

- There are several data gaps which need to be filled including pathogen-specific mortality estimates, and information on morbidity in vulnerable populations such as immunocompromised people, older people and pregnant women.

\section{INTRODUCTION}

Food safety is a global priority. ${ }^{1}$ To have maximum impact, the design and funding of food safety interventions need to take account the overall burden of foodborne disease and the contribution made by each pathogen. Developing better methods for estimating the true burden of foodborne disease has been the focus of international efforts for over a decade. ${ }^{1-8}$ This is problematic for various reasons: people usually present with non-specific symptoms of infectious intestinal disease (IID), only a fraction of cases are confirmed by laboratory testing, and not all are reported to national public health surveillance. IID then needs to be attributed to transmission route (foodborne, waterborne, animal-to-person, person-to-person or environment-to-person), which can be difficult if robust epidemiological information is lacking. In a recent population-based, prospective study in the UK (known as the IID2 study) we found that IID affected around one 
in four people each year $(\sim 17$ million cases in 2009$) .{ }^{9} \mathrm{We}$ used novel methods to estimate, for each pathogen, the proportion of IID attributable to food and the health burden of UK-acquired foodborne disease.

\section{METHODS}

Data sources

The IID studies

Two population-based studies of IID have taken place in the UK (box 1). The first (IID1 study) was conducted in England in 1993-1996, ${ }^{9}$ and the second (IID2 study) took place across the whole of the UK in $2008-2009 .{ }^{10-11}$ Both comprised (1) a prospective cohort study of people living in the community and (2) a prospective study of patients presenting to general practice (GP) with symptoms of IID. Samples were obtained for laboratory testing from symptomatic cases in the cohort and from patients presenting to GP and tested using comprehensive microbiology algorithms. ${ }^{11} 12$ The case definitions were identical in both studies, and incidence rates of IID in the community and GP consultation rates for IID were calculated. Data on healthcare use, captured by questionnaires, gave estimates of hospitalisation rates.

\section{Outbreak surveillance data}

The four UK national surveillance centres provided data on general outbreaks of IID occurring between 1 January 2001 and 31 December $2008(\mathrm{n}=2965$; table 1). There were substantial changes to outbreak reporting in 2009. Prior to 2009 Public Health England (PHE) collected data on all gastrointestinal infection outbreaks no matter what the transmission route was, that is, foodborne, waterborne, person to person, environment to person and animal to person. In 2009 PHE limited the collection of outbreak data on 'non-foodborne outbreaks' to 'gastrointestinal outbreaks including illnesses associated with recreational water exposure, environmental exposure at outdoor events example contact with mud, contact with animals or their faeces and outbreaks of verocytotoxin-producing Escherichia coli (VTEC) mediated through person-to-person transmission'. Thus the data collected from December 2008 represented a subset of outbreaks rather than all outbreaks. This affected the proportion of illnesses assessed as foodborne because the denominators of outbreaks and cases in outbreaks changed substantially as a result of changes

\section{Box 1 Sample sizes in the IID1 and IID2 studies $^{9} 10$}
IID1, England, August 1993-January 1996:
Prospective Cohort Study: N=9776;
General Practice Presentation Study: N=4026.
IID2, UK, April 2008-August 2009:
Prospective Cohort Study: N=7033;
General Practice Presentation Study: N=991.

in reporting definitions. This was particularly problematic for pathogens such as norovirus and Cryptosporidium.

For each outbreak, information was available on the following: outbreak setting, number of cases affected, number of cases hospitalised, main mode(s) of transmission, pathogen identified and, for outbreaks involving contaminated foods, the implicated food vehicle (where this was ascertained). For this study, point source or disseminated outbreaks involving contaminated food, and outbreaks involving contaminated food with subsequent person-to-person transmission, were considered to be foodborne. In total, there were 446 outbreaks involving foodborne transmission that were available for analysis.

\section{Systematic literature review}

We conducted a systematic literature review according to the Preferred Reporting Items for Systematic Reviews and Meta-Analyses (PRISMA) guidelines. ${ }^{13}$ We searched four databases (MEDLINE, EMBASE, Web of Science and FoodBase-the UK Food Standards Agency's research projects database). The full methodology for our systematic review, and a summary of the results have been reported previously. ${ }^{14}$ We also compared the list of articles that we identified through the systematic review with a list of case-control studies included in a separate, independently published review of case-control study methods for enteric infection. ${ }^{15}$ We identified 32 articles published between 1 January 2001 and 31 December 2011 with relevant information that allowed us to determine the percentage of cases of IID attributable to foodborne transmission (see also online supplementary technical appendix). The Bayesian priors were based on uniform distributions, which essentially assumed that any value within a specified range is likely equal. The lower and upper bounds of the distribution were determined by the lowest and highest estimates from studies found in the literature review. So for example, the reported range for foodborne Campylobacter was between $42 \%$ and $80 \%$, and these percentages formed the lower and upper bounds used for the uniform prior (see online supplementary technical appendix).

\section{Modelling approach}

We developed a model to estimate the number of cases, GP consultations and hospital admissions of UK-acquired foodborne disease due to 13 major enteric pathogens: Clostridium perfringens, Campylobacter, E. coli O157, Listeria, Salmonella (non-typhoidal), Shigella, Cryptosporidium, Giardia, adenovirus, astrovirus, norovirus, rotavirus and sapovirus. The basic model was:

$$
\begin{gathered}
\mathrm{F}_{\mathrm{p}}=\mathrm{Nc}_{\mathrm{p}} \pi_{\mathrm{p}} \\
\mathrm{G}_{\mathrm{p}}=\mathrm{Ng}_{\mathrm{p}} \pi_{\mathrm{p}} \\
\mathrm{H}_{\mathrm{p}}=\mathrm{F}_{\mathrm{p}} \gamma_{\mathrm{p}}
\end{gathered}
$$

where $F_{p}, G_{p}$ and $H_{p}$ represent, respectively, the estimated number of UK-acquired foodborne disease cases, GP consultations or hospital admissions for pathogen 
Table 1 Summary of outbreak data for food attribution by pathogen, UK 2001-2008

\begin{tabular}{|c|c|c|c|c|c|c|c|}
\hline \multirow[b]{2}{*}{ Organism } & \multicolumn{3}{|c|}{ Foodborne outbreaks } & \multicolumn{3}{|c|}{$\begin{array}{l}\text { Cases in foodborne } \\
\text { outbreaks }\end{array}$} & \multirow[b]{2}{*}{ Source } \\
\hline & Foodborne & All outbreaks & Per cent & Cases & All cases & Per cent & \\
\hline \multicolumn{8}{|l|}{ Bacteria } \\
\hline C. perfringens & 45 & 60 & 75.0 & 1691 & 1964 & 86.1 & Outbreak surveillance \\
\hline Campylobacter & 31 & 44 & 70.5 & 373 & 761 & 49.0 & Outbreak surveillance \\
\hline E. coli 0157 & 25 & 86 & 29.1 & 564 & 1041 & 54.2 & Outbreak surveillance \\
\hline Listeria & 2 & 2 & 100.0 & 6 & 6 & 100.0 & Outbreak surveillance \\
\hline Salmonella & 266 & 308 & 86.4 & 7128 & 7892 & 90.3 & Outbreak surveillance \\
\hline Shigella & 4 & 11 & 36.4 & 65 & 310 & 21.0 & Outbreak surveillance \\
\hline \multicolumn{8}{|l|}{ Protozoa } \\
\hline Cryptosporidium & 4 & 65 & 6.2 & 415 & 1375 & 30.2 & Outbreak surveillance \\
\hline Giardia & 1 & 7 & 14.3 & 106 & 159 & 66.7 & Outbreak surveillance \\
\hline \multicolumn{8}{|l|}{ Viruses } \\
\hline Adenovirus & - & - & - & - & - & - & No outbreaks reported \\
\hline Astrovirus & 0 & 18 & 0.0 & 0 & 283 & 0.0 & Outbreak surveillance \\
\hline Norovirus & 61 & 2228 & 2.7 & 1500 & 58855 & 2.5 & Outbreak surveillance \\
\hline Sapovirus & - & - & - & - & - & - & No outbreaks reported \\
\hline Rotavirus & 1 & 136 & 0.7 & 30 & 2338 & 1.3 & Outbreak surveillance \\
\hline
\end{tabular}

$\mathrm{p}$ in 2009. $\mathrm{C}_{\mathrm{p}}$ is the UK incidence of IID due to pathogen $p$ and $g_{p}$ is the GP consultation rate for IID due to pathogen $\mathrm{p}$. The constant, $\mathrm{N}$, is the mid-2009 population of the UK. The two parameters, $\pi_{\mathrm{p}}$ and $\gamma_{\mathrm{p}}$, represent, respectively, the proportion of IID cases due to pathogen $\mathrm{p}$ that are transmitted through food, and the proportion of cases due to pathogen $\mathrm{p}$ that are hospitalised. We assumed that foodborne cases were equally likely to consult a GP or be hospitalised as non-foodborne cases.

We used various data sources to inform model parameters. The data available for each pathogen are summarised in the online supplementary technical appendix tables A1-A3. We used two modelling approaches: a Monte Carlo simulation approach and a Bayesian approach. In the Monte Carlo approach, the parameters $\pi_{\mathrm{p}}$ and $\gamma_{\mathrm{p}}$ were defined by $\beta$ distributions fitted to empirical bootstrap samples of UK outbreak data; in the Bayesian approach, these parameters were modelled as binomial quantities and given priors informed by published studies and hospitalisation data from previous studies in the UK. Model details are given in the online supplementary technical appendix.

\section{Pathogen-specific rates of IID $\left(c_{p}, \gamma_{p}\right)$}

We obtained data from the IID2 study on population incidence and GP consultation rates for IID, and their associated uncertainty, for the above pathogens. ${ }^{10}$ For Shigella, no cases were found in IID2 so we applied the reporting ratio from IID1 (the ratio of community cases to laboratory-confirmed cases reported to national surveillance) to the number of cases reported in 2009 and divided this by the mid-2009 UK population to obtain the overall shigellosis rate. ${ }^{11}$ Similarly, we estimated GP consultation rates by applying the reporting ratio from IID1 (the ratio of GP consultations to laboratory- confirmed cases reported to national surveillance) to the number of laboratory reports in 2009. We accounted for uncertainty in incidence estimates by sampling 100000 times from the distribution of reporting ratios estimated in IID1. For Listeria, no incidence data were available from IID1 or IID2 so we used the number of laboratory reports for listeriosis in 2009 as a conservative population incidence estimate.

\section{Proportion of cases transmitted through food $\left(\pi_{\mathrm{p}}\right)$}

Estimating the proportion of cases transmitted through food

We used data on outbreaks reported to national surveillance systems between January 2001 and December 2008 to estimate the proportion of cases transmitted through food. For each pathogen, we computed empirical estimates for $\pi_{\mathrm{p}}$ by obtaining 4999 bootstrap samples of the proportion of cases in outbreaks that resulted from foodborne transmission. We then fitted a $\beta$ function to the resulting distribution using maximum likelihood. For Cryptosporidium and Giardia, this approach gave an unrealistically high estimate for the proportion of cases transmitted through food because, of the few outbreaks that were reported, those involving foodborne transmission were larger. For these two pathogens, we used instead the proportion of outbreaks that were foodborne as an estimate of $\pi_{\mathrm{p}}$, as was done in a previous study. ${ }^{5}$ For adenovirus and sapovirus, for which no outbreaks were reported, we used parameters derived from analysis of rotavirus and norovirus outbreaks respectively. For pathogens for which all outbreaks or no outbreaks were foodborne, we specified limits to the fitted $\beta$ distributions as described in the online supplementary technical appendix. The $\mathrm{a}$ and $\mathrm{b}$ parameters from the fitted $\beta$ distributions were then used in the Monte Carlo simulations (see Model 1 below). 
Prior distributions for the proportion of cases transmitted through food $\left(\pi_{p}\right)$

We obtained prior distributions for the $\pi_{\mathrm{p}}$ parameters from the systematic literature review. We divided the retrieved articles into two categories: food attribution studies (Group A) and others (Group B). In Group A studies the proportion of cases transmitted through food was estimated for several pathogens, through expert elicitation or retrospective data reviews. Group B were primarily pathogen-specific case-control studies or studies using microbiological typing for source attribution. For Group A and Group B studies, we defined uniform distributions for $\pi_{\mathrm{p}}$, based on the minimum and maximum estimates of the proportion of cases transmitted through food in these studies, for pathogens with at least two published studies. Where the observed proportion from outbreak data fell outside the limits of this uniform distribution, we arbitrarily allowed the lower or upper limit of the distribution to extend by 0.1 beyond the observed value.

\section{Pathogen-specific hospitalisation $\left(\gamma_{p}\right)$}

Data on hospitalisations were available only for outbreaks reported in England and Wales. For each reported outbreak, excluding those in hospitals or residential institutions, we computed the proportion of cases hospitalised by causative organism. We based hospitalisation estimates on all outbreaks with the available data, as we found no major differences in hospitalisation between foodborne and other outbreaks. To account for uncertainty in these parameters, we fitted $\beta$ distributions to bootstrapped data as detailed above for $\pi_{\mathrm{p}}$, but additionally weighting by outbreak size (see online supplementary technical appendix). For adenovirus and sapovirus, we used parameters derived from analysis of rotavirus and norovirus outbreaks, respectively. Bootstrap estimates with fitted $\beta$ distributions by pathogen are shown in the online supplementary technical appendix.

\section{Prior distributions for pathogen-specific hospitalisation $\left(\gamma_{p}\right)$}

We used pathogen specific, $\beta$-distributed priors for $\gamma_{p}$. The $\beta$ parameters were informed by an analysis of hospitalisation data from the IID1 and IID2 studies (see online supplementary figure A1 and technical appendix).

Estimating food-related IID cases, GP consultations and hospitalisations $\left(\mathrm{F}_{\mathrm{p}}, \mathrm{G}_{\mathrm{p}}, \mathrm{H}_{\mathrm{p}}\right)$. We obtained estimates of the number of foodborne cases, GP consultations and hospitalisations using three different approaches. In Model 1, we used the Monte Carlo simulation to draw values at random from each parameter distribution. In Model 2, we used a Bayesian approach that included parameters for the prior distributions of $\gamma_{p}$ from the IID1 and IID2 studies and for $\pi_{\mathrm{p}}$ from Group A studies as described above. These priors were used, together with the outbreak data, to obtain posterior distributions for these parameters, which were then used in the model. This model could not be applied to sapovirus, because none of the identified studies had information about this pathogen. Model 3 had the same structure as Model 2, except that Bayesian priors for $\pi_{\mathrm{p}}$ from Group B studies were used instead. This model was applied to Campylobacter, E. coli O157, Listeria and Salmonella, for which sufficient data from published studies were available. A full description of model parameters is given in the online supplementary technical appendix.

For each model, we carried out 100000 simulations, discarding the first $10 \%$ and retaining the model outputs for every 10th simulation. We checked model convergence graphically by plotting parameter values over time to verify adequate mixing, plotting autocorrelograms and comparing density plots for outcome variables by tertile of the simulation chain. We summarised model outputs using the median and central $95 \%$ of the posterior distributions to obtain point estimates and $95 \%$ credible intervals (CrI) for the number of food-related cases, GP consultations and hospitalisations by pathogen. We conducted the analyses using Stata V.12.1, WinBUGS and Microsoft Excel software. We used the winbugsfromstata module in Stata to carry out the simulations. ${ }^{16}$

\section{Ethical considerations}

An Ethics Committee favourable opinion was not required. These were secondary analyses of previously collected, publicly available data. All data sets used were completely anonymous and there was no risk of disclosure of personal data.

\section{RESULTS}

Proportion of cases attributable to foodborne transmission

Table 1 summarises the outbreak data used for estimating the proportion of cases due to foodborne transmission from outbreak data. The identified studies used to inform Bayesian uniform priors are summarised in the online supplementary technical appendix tables A2 and A3. Figure 1 shows the empirical bootstrap distributions for the estimated proportion of cases due to foodborne transmission based on outbreak data. For most pathogens, the $\beta$ distribution provided a reasonable fit to the bootstrapped distribution, with the exception of Giardia, for which data were sparse, and rotavirus, for which the estimated proportion foodborne transmission was very small. Salmonella and C. perfringens had the largest estimated proportion of cases attributable to foodborne transmission, each $\sim 90 \%$. Around $50 \%$ of Campylobacter and E. coli $\mathrm{O} 157$ cases were estimated to result from foodborne transmission, although there was considerable uncertainty in these estimates as evidenced by the long tails in these distributions. Foodborne transmission accounted for $<5 \%$ of norovirus cases, while $\sim 65 \%$ of Giardia cases, $30 \%$ of Cryptosporidium cases and $20 \%$ of Shigella cases were food related.

\section{Proportion of cases hospitalised}

Table 2 summarises the data sources used to inform hospitalisation parameters. Figure 2 shows the estimated 

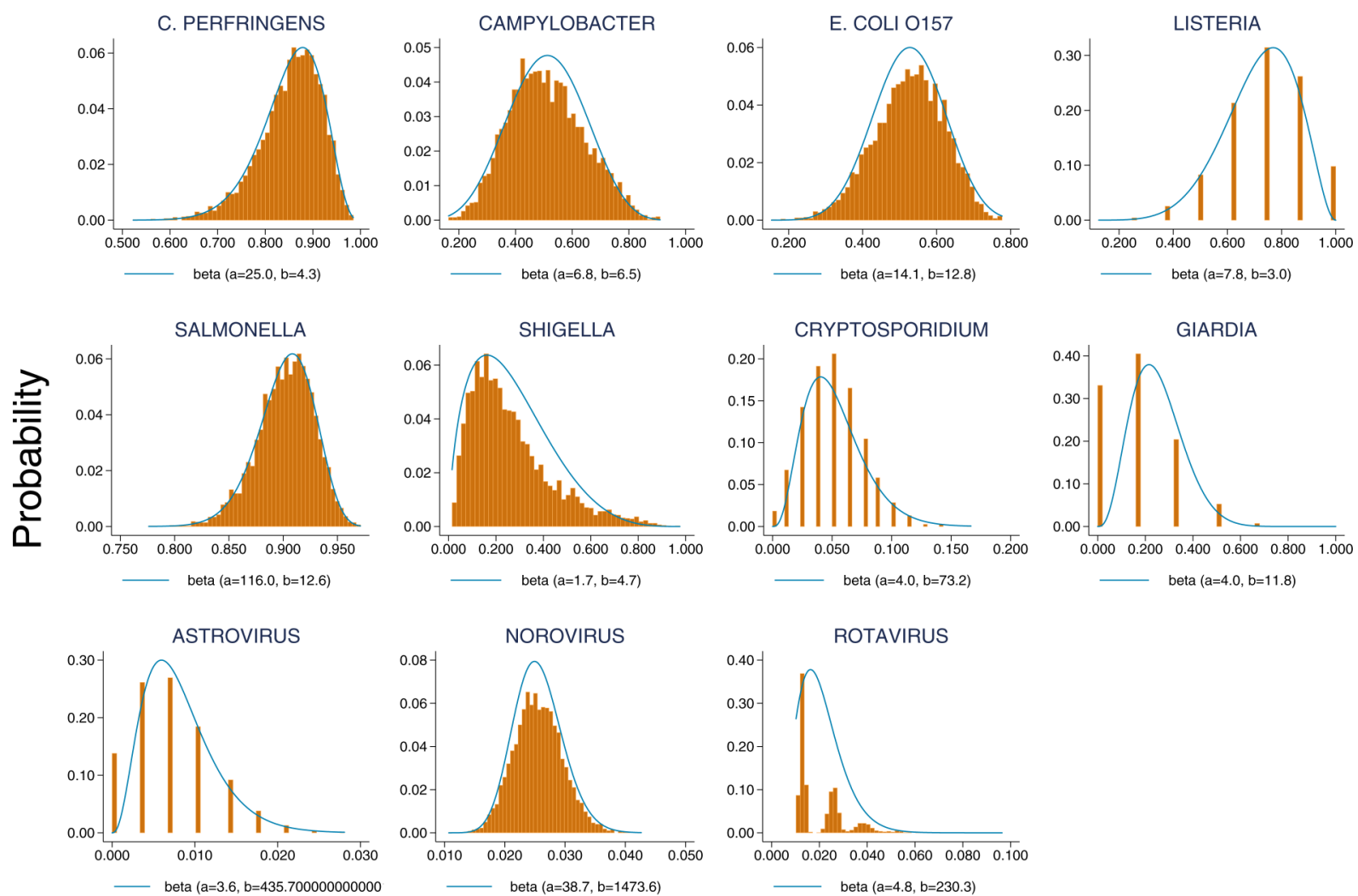

Proportion of cases foodborne

Figure 1 Empirical bootstrap distributions for the estimated proportion of cases due to foodborne transmission based on outbreak data.

hospitalisation proportions in reported outbreaks by pathogen, based on the medians of $\beta$ distributions fitted to outbreak data. Hospitalisation was particularly high for E. coli 0157 (23\%). In contrast, $<2 \%$ of cases due to C. perfringens, Campylobacter, Giardia, norovirus and rotavirus were hospitalised.

Cases, GP consultations and hospital admissions attributable to foodborne transmission (Model 1)

Table 3 presents estimates of food-related cases, GP consultations and hospital admissions in 2009 from 100000 Monte Carlo simulations. Campylobacter was the most common foodborne pathogen, accounting for 286000 food-related cases (95\% CrI 131 105-532 400) and 39750 GP consultations (95\% CrI 18 890-69 540), but ranked third as a cause of food-related hospital admissions (1376 admissions) behind Salmonella (2536 admissions) and E. coli $\mathrm{O} 157$ (2141 admissions). Foodborne norovirus accounted for 3240 GP consultations (95\% CrI 1985-5162), but fewer than 500 hospital admissions. Similarly, other pathogens such as $C$. perfringens and a number of the viruses, while contributing large numbers of cases and GP consultations, were responsible for a modest number of food-related hospital admissions. It should be noted, however, that there was a large degree of uncertainty around these estimates, as demonstrated by the wide $95 \% \mathrm{CrI}$.

\section{Cases, GP consultations and hospital admissions} attributable to foodborne transmission (Models 2 and 3) Estimates of food-related cases, GP consultations and hospital admissions based on the Bayesian approach used in Model 2 are presented in table 4. Campylobacter was the most common foodborne pathogen, causing 280400 (95\% CrI 182 503-435 693) food-related cases and 38860 (95\% CrI 27 160-55 610) GP consultations annually. Despite this, there were only 562 (95\% CrI 189-1330) Campylobacter-related hospital admissions. Salmonella caused the largest number of hospitalisations, an estimated 2490 admissions (95\% CrI 607-9631), closely followed by E. coli $\mathrm{O} 157$ with 2233 admissions (95\% CrI 170-32 159). Other common causes of foodborne disease included C. perfringens, with an estimated 79570 cases annually (95\% CrI 30 700-211 298), and norovirus with 74100 cases (95\% CrI 61 150-89 660). For Model 2, there were insufficient data from the studies we identified to enable estimation of foodborne sapovirus. For Campylobacter, E. coli O157, Listeria and Salmonella, further estimates from Model 3 are presented in table 5. The estimates from the three different models are compared in figure 3A-C. 


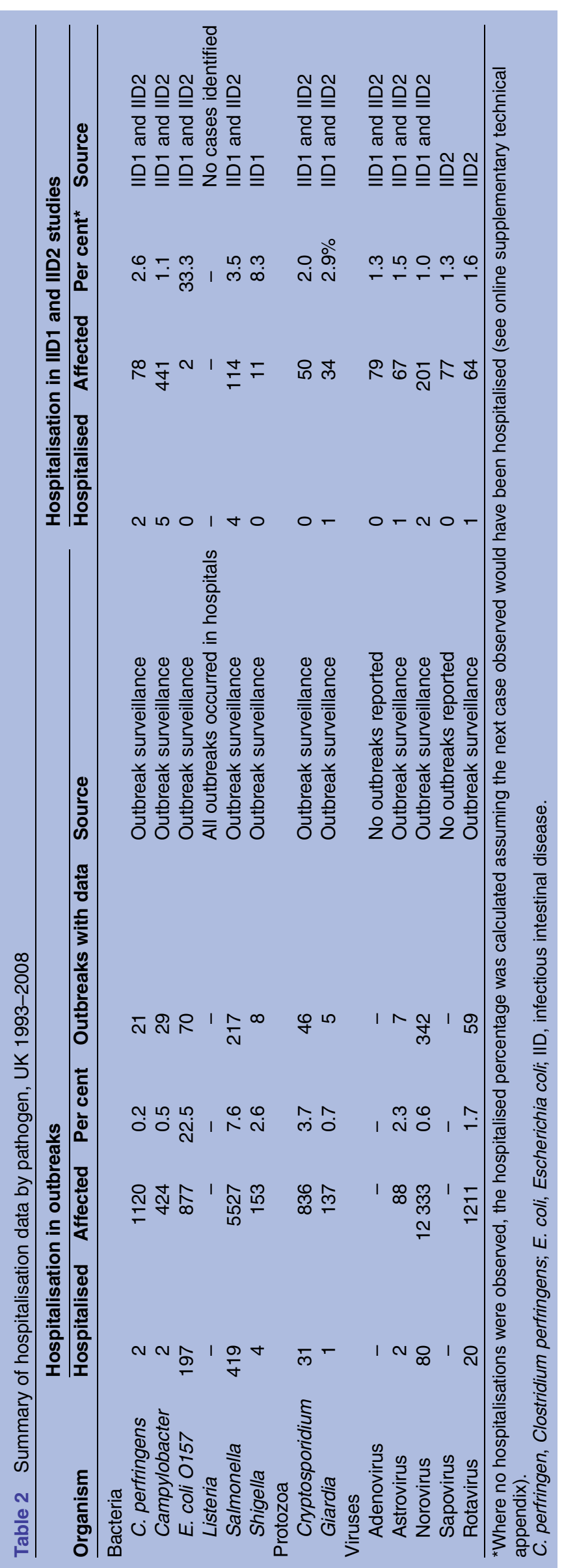

Comparing the models

In general, the results from all three approaches were similar for food-related cases and GP consultations. For most organisms, the Bayesian estimates from Model 2 benefited from greater precision. There were differences in the number of food-related hospital admissions estimated by the Monte Carlo and Bayesian approaches for some organisms, notably Campylobacter, rotavirus, adenovirus and astrovirus. The differences reflect discordance between outbreak data and data from the IID studies in terms of the hospitalisation rate for these organisms. Where differences were observed, the Bayesian approach gave more conservative estimates of the number of food-related hospital admissions.

For the four pathogens with sufficient data from the literature review to generate estimates from Model 3 (Campylobacter, E. coli O157, Listeria and Salmonella), estimates were similar to those from Model 2; however, Listeria estimates carried greater uncertainty, because of wide disagreement between the two identified studies regarding the proportion of listeriosis attributable to foodborne transmission. It was impossible to calculate listeriosis hospitalisations because all reported Listeria outbreaks occurred in hospitals.

\section{DISCUSSION}

To the best of our knowledge this is the first study to incorporate empirical data and prior information from a systematic review using Bayesian methodology for estimating the proportion of IID that is transmitted through contaminated food. Campylobacter is the most common foodborne pathogen in the UK, causing between 182503 and 435693 food-related cases and between 27160 and 55610 GP consultations annually (based on Model 2 results). Despite this, the number of Campylobacter-related hospital admissions is comparatively small, reflecting a generally lower level of acute disease severity compared with other pathogens. In contrast, Salmonella and E. coli O157 cause the largest number of hospitalisations, an estimated 2490 and 2233 admissions respectively (Model 2 ), although uncertainty around these estimates is high. Other common causes of foodborne illness include $C$. perfringens, responsible for nearly 80000 cases annually and norovirus, responsible for nearly 75000 cases. Other viral agents rank lower as causes of foodborne illness.

Our analysis updates previous estimates for England and Wales in 2000 and expands on them by accounting for uncertainty. ${ }^{5}$ Owing to substantial differences in the analyses, the two sets of estimates are not directly comparable. Other studies investigating the burden of foodborne illness caused by a wide range of pathogens have been carried out in Australia, the USA and the

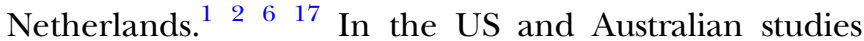
norovirus was one of the commonest causes of foodborne disease. In the US study, it was also the second most common cause of food-related hospital admissions. Approximately one-quarter of norovirus IID cases in 
Figure 2 Estimated

hospitalisation proportions in reported outbreaks by pathogen, based on the medians of $\beta$ distributions fitted to outbreak data.

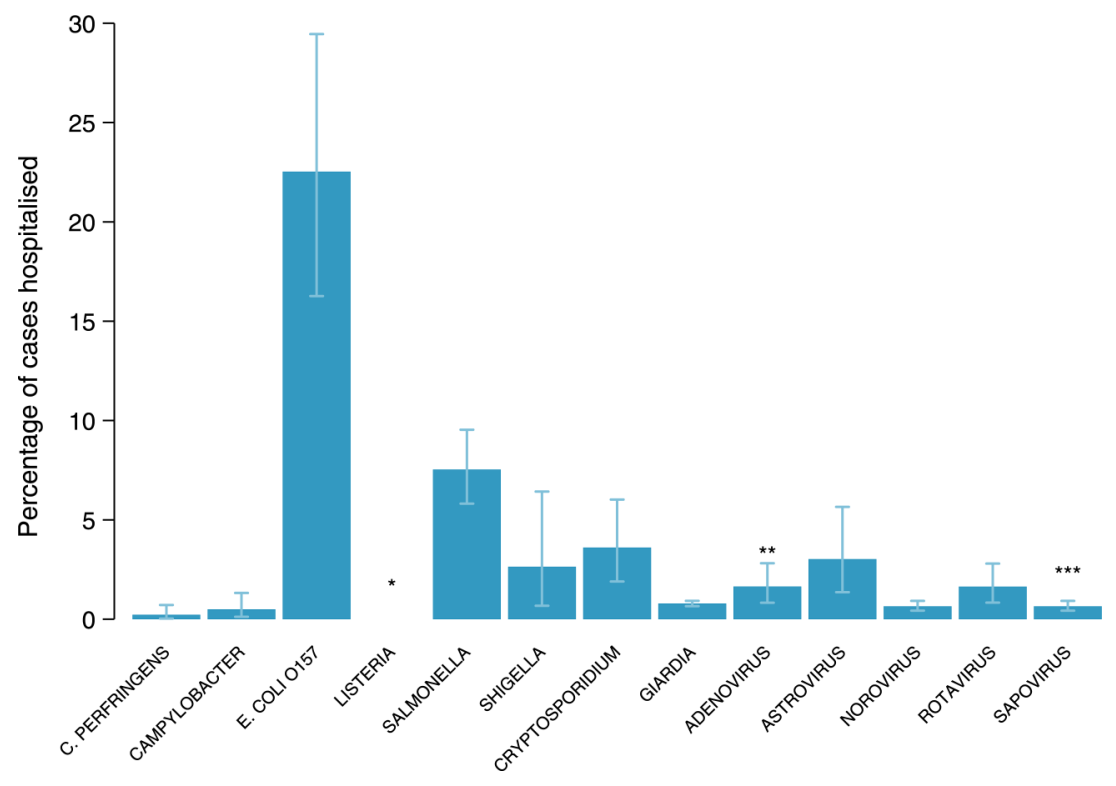

those two studies were attributed to foodborne transmission, whereas our estimate for the UK is $<5 \%$. A likely reason for this discrepancy is the definitions of outbreaks that are incorporated in the various modelling studies. Some data sets contain only outbreaks transmitted through food while others, like ours (until 2009), contained all outbreaks of IID no matter what the route of transmission. This means that the proportion of norovirus cases transmitted through food is likely to be overestimated in data sets that contain only outbreaks transmitted through food.

A major strength of our analysis is the availability of directly observed, pathogen-specific incidence data from the recent IID2 study in the UK, ${ }^{10}$ which precludes the need to adjust for underascertainment and requires fewer assumptions about healthcare usage. The use of methods to account fully for parameter uncertainties is an additional strength, and is useful for highlighting areas where data are sparse. This is particularly true for hospitalisation estimates, for which there is a dearth of reliable data. We investigated other sources of hospitalisation data, such as electronic records of inpatient admissions. However, these data lack specific diagnostic codes for certain key pathogens, including E. coli $\mathrm{O} 157$, and a large fraction of admissions are classified under nonspecific diagnostic codes. We therefore used outbreak data to estimate hospitalisation. A potential limitation is that severe cases requiring hospitalisation might be more

Table 3 Estimates of food-related cases, GP consultations and hospitalisations by pathogen, UK 2009 (Model 1)

\begin{tabular}{|c|c|c|c|c|c|c|}
\hline Organism & Cases & (95\% Crl) & GP consultations & (95\% Crl) & $\begin{array}{l}\text { Hospital } \\
\text { admissions }\end{array}$ & (95\% Crl) \\
\hline \multicolumn{7}{|l|}{ Bacteria } \\
\hline C. perfringens & 79165 & (29 310-208 688) & 12610 & (5707-27 890) & 165 & $(20-843)$ \\
\hline Campylobacter & 286000 & $(131$ 105-532 400) & 39750 & (18 890-69 540) & 1376 & (289-4607) \\
\hline E. coli $\mathrm{O} 157$ & 9536 & (644-146 495) & 324 & (36-2973) & 2141 & (143-33 237) \\
\hline Listeria & 169 & $(100-215)$ & 169 & $(100-215)$ & - & - \\
\hline Salmonella & 33640 & (8286-135 798) & 10030 & (4019-24 299) & 2536 & $(608-10400)$ \\
\hline Shigella & 1274 & (90-11990) & 684 & $(84-2145)$ & 32 & $(2-378)$ \\
\hline \multicolumn{7}{|l|}{ Protozoa } \\
\hline Cryptosporidium & 2035 & (354-10 129) & 588 & $(140-2010)$ & 72 & $(12-395)$ \\
\hline Giardia & 11250 & (2239-52 878) & 1322 & (286-4960) & 88 & $(17-415)$ \\
\hline \multicolumn{7}{|l|}{ Viruses } \\
\hline Adenovirus & 11920 & (3706-28 909) & 987 & (293-2536) & 191 & $(51-559)$ \\
\hline Astrovirus & 2362 & (594-7180) & 180 & $(41-576)$ & 70 & $(15-262)$ \\
\hline Norovirus & 73420 & (50 320-104 000) & 3240 & (1985-5162) & 470 & $(270-779)$ \\
\hline Rotavirus & 14850 & $(4698-35330)$ & 1603 & $(494-3856)$ & 237 & $(64-688)$ \\
\hline Sapovirus & 40770 & (26 661-60 230) & 2457 & (1496-3947) & 261 & (145-445) \\
\hline Total & 566391 & & 73944 & & 7639 & \\
\hline
\end{tabular}


Table 4 Estimates of food-related cases, GP consultations and hospitalisations by pathogen, UK 2009 (Model 2)

\begin{tabular}{|c|c|c|c|c|c|c|}
\hline Organism & Cases & (95\% Crl) & $\begin{array}{l}\text { GP } \\
\text { consultations }\end{array}$ & (95\% Crl) & $\begin{array}{l}\text { Hospital } \\
\text { admissions }\end{array}$ & (95\% Crl) \\
\hline \multicolumn{7}{|l|}{ Bacteria } \\
\hline C. perfringens & 79570 & (30 700-211 298) & 12680 & $(6072-27040)$ & 186 & (38-732) \\
\hline Campylobacter & 280400 & (182 503-435 693) & 38860 & (27 160-55 610) & 562 & $(189-1330)$ \\
\hline E. coli $\mathrm{O} 157$ & 9886 & (748-142 198) & 342 & $(37-3030)$ & 2233 & (170-32 159) \\
\hline Listeria & 183 & $(161-217)$ & 183 & $(161-217)$ & - & - \\
\hline Salmonella & 33130 & (8178-128 195) & 10060 & $(4137-24710)$ & 2490 & $(607-9631)$ \\
\hline Shigella & 1204 & (181-8142) & 602 & (341-1060) & 33 & $(4-270)$ \\
\hline \multicolumn{7}{|l|}{ Protozoa } \\
\hline Cryptosporidium & 2773 & $(562-12200)$ & 800 & $(233-2386)$ & 94 & $(18-436)$ \\
\hline Giardia & 7877 & (1467-36 059) & 883 & (197-3288) & 47 & $(4-332)$ \\
\hline \multicolumn{7}{|l|}{ Viruses } \\
\hline Adenovirus & 8253 & $(4734-13780)$ & 677 & (345-1278) & 62 & $(30-118)$ \\
\hline Astrovirus & 3470 & (1368-9991) & 262 & (93-812) & 11 & (3-42) \\
\hline Norovirus & 74100 & $(61$ 150-89 660) & 3276 & (2240-4729) & 332 & $(248-440)$ \\
\hline Rotavirus & 10295 & $(6049-16$ 730) & 1102 & $(629-1870)$ & 95 & $(48-177)$ \\
\hline Sapovirus* & - & - & - & - & - & - \\
\hline TOTAL & 511141 & & 69727 & & 6145 & \\
\hline
\end{tabular}

${ }^{*}$ For sapovirus, no data were identified in the literature review on the proportion of cases attributable to food, so this model could not be applied. C. perfringens, Clostridium perfringens; E. coli, Escherichia coli; GP, general practice.

reliably recorded in outbreak reports, whereas milder cases might be missed. There might genuinely be higher hospitalisation rates in outbreaks than sporadic cases because of higher dose exposures or different populations might be affected in outbreaks. Alternatively, outbreaks with more hospitalised cases might be more likely to be investigated and reported. This would tend to overestimate hospitalisation rates. Such a bias is possible in the E. coli $\mathrm{O} 157$ data, where estimates for hospitalisations were considerably higher than for GP consultations. Alternatively, the severity of this disease could mean that cases are admitted directly to hospital without first consulting a GP. Our Bayesian models additionally incorporated prior information on hospitalisation rates from IID1 and IID2. For most pathogens, the two types of models gave similar results. However, the number of hospitalisations in both sets of data were small, reflected in the large degree of uncertainty in the estimates. For rotavirus and astrovirus, the Bayesian model gave somewhat lower estimates of hospital admissions, which might indicate that hospitalisations for these two pathogens are over-reported in outbreak data or that they were underascertained in the IID studies. Additionally, outbreaks might occur in specific age groups or individuals with underlying conditions or be due to high-dose exposure. Outbreak reports, however, contain limited information on the populations affected.

Using outbreak data to attribute cases of IID to foodborne transmission relies on certain assumptions, principally that outbreak cases reflect the epidemiology in the wider community. Another potential limitation is that there might be a bias towards investigation or reporting of foodborne outbreaks compared with outbreaks transmitted through other routes, like person-to-person transmission. This, however, does not seem to be the case: there has been a gradual decrease in the proportion of reported outbreaks involving foodborne transmission, which reflects both a reduction in incidence of certain foodborne pathogens, particularly Salmonella, and greater investigation of outbreaks in other settings, particularly viral outbreaks in hospitals and residential institutions. $^{18} 19$

Our study focused on foodborne illness burden in the general UK population. Some pathogens, however, are a

Table 5 Estimates of food-related cases, GP consultations and hospitalisations by pathogen, UK 2009 (Model 3)

\begin{tabular}{lrlllll}
\hline Organism & Cases & $\mathbf{( 9 5 \% ~ C r l )}$ & GP consultations & $\mathbf{( 9 5 \% ~ C r l )}$ & Hospital admissions & $\mathbf{( 9 5 \% ~ C r l ) ~}$ \\
\hline Campylobacter & 279900 & $(183100-433098)$ & 38820 & $(27010-55580)$ & 561 & $(189-1343)$ \\
E. coli O157 & 9536 & $(644-146495)$ & 324 & $(36-2973)$ & 2141 & $(143-33237)$ \\
Listeria & 166 & $(92-214)$ & 166 & $(92-214)$ & - & - \\
Salmonella & 33130 & $(8178-128195)$ & 10060 & $(4137-24710)$ & 2490 & $(607-9631)$ \\
TOTAL & 322732 & & 49370 & & 5192 & \\
\hline
\end{tabular}

${ }^{*}$ For Listeria, the number of hospital admissions could not be calculated, as all reported outbreaks occurred in hospitals.

E. coli, Escherichia coli; GP, general practice. 

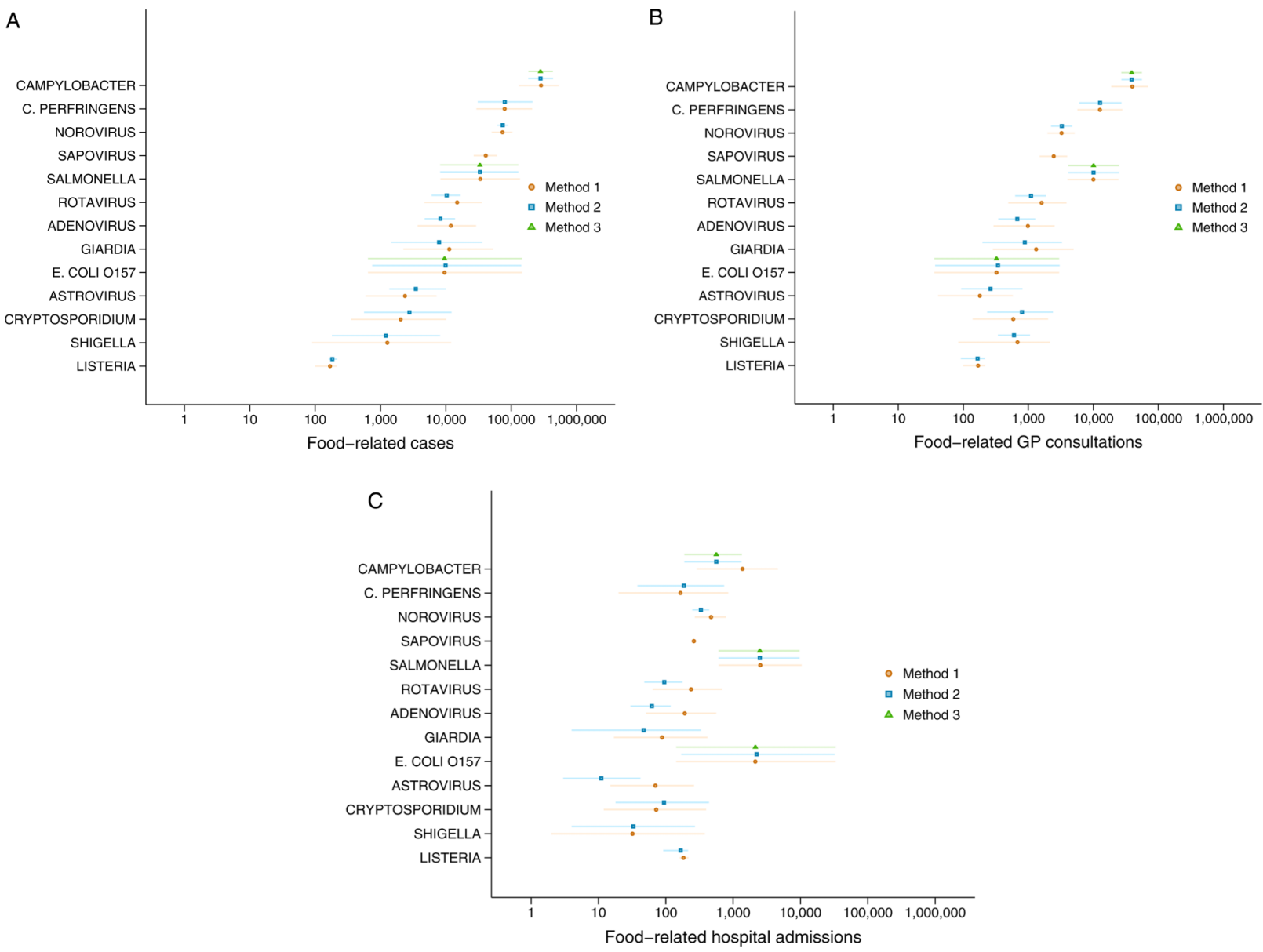

Figure 3 (A) Comparison of estimates from Monte Carlo and Bayesian approaches_food-related cases, UK 2009 (Model 1: Monte Carlo simulation approach; Model 2: Bayesian approach using data from published food attribution studies; Model 3: Bayesian approach using data from published pathogen-specific studies (error bars show $95 \% \mathrm{Crl}$ ). (B) Comparison of estimates from Monte Carlo and Bayesian approaches_food-related general practice consultations, UK 2009 (Model 1: Monte Carlo simulation approach; Model 2: Bayesian approach using data from published food attribution studies; Model 3: Bayesian approach using data from published pathogen-specific studies (error bars show 95\% Crl)). (C) Comparison of estimates from Monte Carlo and Bayesian approaches_food-related hospital admissions, UK 2009 (Model 1: Monte Carlo simulation approach; Model 2: Bayesian approach using data from published food attribution studies; Model 3: Bayesian approach using data from published pathogen-specific studies (error bars show 95\% Crl)). C. perfringens, Clostridium perfringens, E. coli, Escherichia coli.

particular problem among certain high-risk groups, such as Listeria among immunocompromised patients and pregnant women and rotavirus among children under 5 years. Our analysis was not designed to estimate burden in these subgroups, because our data sources contain limited information on these groups, and the size of some of these high-risk populations is uncertain. However, further studies to estimate burden in these groups is warranted.

We were unable to include other relevant pathogens such as toxoplasmosis, hepatitis $\mathrm{A}$, hepatitis $\mathrm{E}$ and non-O157 VTEC in our analysis, due to a lack of relevant data in the UK. In a Dutch study Toxoplasma gondii caused the highest foodborne disease burden as measured by disability-adjusted life years, reflecting the importance of congenital toxoplasmosis. ${ }^{16}$

Our modelling approach meant we could use data from various sources to incorporate the best available information from the UK and elsewhere. Comparing models with and without prior information indicates where there is disagreement between data sources and enables uncertainty in all the relevant parameters to be accounted for. Uncertainty in these models reflects not simply statistical uncertainty in individual parameters, but disagreement between data sources and availability of information from previous studies. Information from previous studies on the proportion of IID transmitted through food was captured using Bayesian uniform priors. This is probably conservative, as it presupposes that every value within the specified limits is likely equal. For most pathogens, however, the number of available studies was small and using more informative priors was difficult to justify. The exception was Campylobacter, for which 14 studies contained relevant data. Even so, using data from risk factor studies presents problems in interpretation. Study design, methods and risk factors 
investigated varied widely. Consequently, variability between studies in the importance of food-related risk factors is high. The choice of Bayesian priors in estimation is necessarily a subjective process, as it depends on analysts' confidence in the available information. Establishment of a process to develop greater international consensus on the choice of priors for individual pathogens could help to refine future estimates. Better baseline estimates would also inform predictions of the likely increase in foodborne disease due to climate change. ${ }^{20}$

We did not estimate deaths attributable to foodborne illness, due to the lack of reliable data sources on pathogen-specific mortality rates. Death certificates rarely provide information on specific gastrointestinal pathogens, while deaths in outbreaks are rare and may not be recorded if they occur sometime after the event. More generally, mortality estimates would be difficult to interpret. Deaths attributed to foodborne disease are not necessarily the same as preventable deaths. More focused epidemiological studies on mortality following IID would be helpful.

Our estimates measure foodborne disease burden only in the acute phase of illness. For some pathogens, the long-term consequences of illness can add considerably to their burden, for example E. coli O157-associated haemolytic uraemic syndrome and Campylobacter-associated Guillain-Barré syndrome. ${ }^{21} 22$ Moreover, our estimates are based only on the number of cases of illness, and take no account of the consequences of illness in different sectors of the population. Further studies using additional measures of disease burden and taking into account long-term health consequences are therefore required.

Modelling is not necessarily a substitute for acquiring good quality primary data but it is very useful for pointing to important data gaps and major areas of uncertainty where primary data collection might be focused.

Controlling foodborne disease is an important policy issue. Given the burden of illness caused, there needs to be a continued focus on reducing illness due to Campylobacter, Salmonella, C. perfringens and norovirus.

\section{Author affiliations \\ ${ }^{1}$ University of Liverpool Institute of Infection and Global Health, Liverpool, UK ${ }^{2}$ NIHR Health Protection Research Unit in Gastrointestinal Infections, The Farr Institute@HeRC, University of Liverpool, Liverpool, UK \\ ${ }^{3}$ Department of Infectious Disease Epidemiology, London School of Hygiene and Tropical Medicine, London, UK \\ ${ }^{4}$ Department of Public Health and General Practice, Norwegian University of Science and Technology, Trondheim, Norway \\ ${ }^{5}$ Department of Gastrointestinal, Emerging \& Zoonotic Infections, Public Health England Centre for Infectious Disease Surveillance and Control, London, UK \\ ${ }^{6}$ Institute of Primary Care and Public Health, Cardiff University, Cardiff, UK ${ }^{7}$ Saw Swee Hock School of Public Health, National University of Singapore, Singapore, Singapore}

Acknowledgements The authors would like to thank John Cowden and Brian Smyth for expert interpretation of outbreak data and the information scientists at the four UK national surveillance centres for providing the outbreak data on which some of the analyses are based.
Collaborators Additional members are Paul Cook, John M Cowden, Kathryn A Jackson, Brian Smyth.

Contributors The study was conceived by CCT and SJO; TLL conducted the systematic literature review; GKA and MRE provided expert interpretation of the outbreak surveillance data used in the analysis; CCT conducted the analysis; SJO and CCT wrote the initial draft manuscript; SJO, TLL, GKA, MRE and CCT interpreted the results and made substantial contributions to revising the manuscript.

Funding This work was funded by the Food Standards Agency grant number FS231043, awarded to SJ0 as Project Lead Contractor. SJ0 is also supported by the National Institute for Health Research Health Protection Research Unit in Gastrointestinal Infections at the University of Liverpool (Grant number NIHR HPRU 2012-10038). The research was partly funded by the National Institute for Health Research Health Protection Research Unit (NIHR HPRU) in Gastrointestinal Infections at the University of Liverpool in partnership with Public Health England (PHE), University of East Anglia, University of Oxford and the Institute of Food Research.

Disclaimer The views expressed are those of the authors and not necessarily those of the NHS, the NIHR, the Department of Health or PHE.

\section{Competing interests None declared.}

Provenance and peer review Not commissioned; externally peer reviewed.

Data sharing statement Data from the IID1 study are available from the UK Data Service (http://ukdataservice.ac.uk/; doi: 10.5255/UKDA-SN-4092-1). Data from the IID2 study are available from the UK Data Service (http://dx.doi. org/10.5255/UKDA-SN-7820-1). Anonymous outbreak surveillance data are available on request from Public Health England (eFOSS, https://bioinfosecure. phe.org.uk/efoss), Health Protection Scotland and the Public Health Agency of Northern Ireland. Data from the literature review are in the public domain and available as cited in the main manuscript and/or online supplementary technical appendix.

Open Access This is an Open Access article distributed in accordance with the terms of the Creative Commons Attribution (CC BY 4.0) license, which permits others to distribute, remix, adapt and build upon this work, for commercial use, provided the original work is properly cited. See: http:// creativecommons.org/licenses/by/4.0/

\section{REFERENCES}

1. Kuchenmüller T, Abela-Ridder B, Corrigan T, et al. World Health Organization initiative to estimate the global burden of foodborne diseases. Rev Sci Tech 2013;32:459-67.

2. Scallan E, Hoekstra RM, Angulo FJ, et al. Foodborne illness acquired in the United States-major pathogens. Emerging Infect Dis 2011;17:7-15.

3. Scallan E, Griffin PM, Angulo FJ, et al. Foodborne illness acquired in the United States-unspecified agents. Emerging Infect Dis 2011;17:16-22.

4. Mead PS, Slutsker L, Dietz V, et al. Food-related illness and death in the United States. Emerging Infect Dis 1999;5:607-25.

5. Adak GK, Long SM, O'Brien SJ. Trends in indigenous foodborne disease and deaths, England and Wales: 1992 to 2000. Gut 2002;51:832-41.

6. Hall G, Kirk MD, Becker N, et al. Estimating foodborne gastroenteritis, Australia. Emerging Infect Dis 2005;11:1257-64.

7. Vaillant $\mathrm{V}$, de Valk $\mathrm{H}$, Baron $\mathrm{E}$, et al. Foodborne infections in France. Foodborne Pathog Dis 2005;2:221-32.

8. Flint JA, Van Duynhoven YT, Angulo FJ, et al. Estimating the burden of acute gastroenteritis, foodborne disease, and pathogens commonly transmitted by food: an international review. Clin Infect Dis 2005;41:698-704

9. Tam CC, Rodrigues LC, Viviani L, et al, IID2 Study Executive Committee. Longitudinal study of infectious intestinal disease in the UK (IID2 study): incidence in the community and presenting to general practice. Gut 2012;61:69-77.

10. Wheeler JG, Sethi D, Cowden JM, et al. Study of infectious intestinal disease in England: rates in the community, presenting to general practice, and reported to national surveillance. The Infectious Intestinal Disease Study Executive. BMJ 1999;318:1046-50.

11. O'Brien SJ, Rait G, Hunter PR, et al. Methods for determining disease burden and calibrating national surveillance data in the 
United Kingdom: the second study of infectious intestinal disease in the community (IID2 study). BMC Med Res Methodol 2010;10:39.

12. Tompkins DS, Hudson MJ, Smith HR, et al. A study of infectious intestinal disease in England: microbiological findings in cases and controls. Commun Dis Public Health 1999;2:108-13.

13. Liberati A, Altman DG, Tetzlaff J, et al. The PRISMA statement for reporting systematic reviews and meta-analyses of studies that evaluate health care interventions: explanation and elaboration. J Clin Epidemiol 2009;62:e1-34.

14. Tam CC, Larose T, O'Brien SJ. Costed extension to the second study of infectious intestinal disease in the community: identifying the proportion of foodborne disease in the UK and attributing foodborne disease by food commodity. UK: Food Standards Agency, 2014. Report No.: Project B18021 (FS231043). http://www. food.gov.uk/sites/default/files/IID2\%20extension\%20report\%20-\% 20FINAL\%2025\%20March\%202014_0.pdf (accessed 31 Jul 2015).

15. Fullerton KE, Scallan E, Kirk MD, et al. Case-control studies of sporadic enteric infections: a review and discussion of studies conducted internationally from 1990 to 2009. Foodborne Pathog Dis 2012;9:281-92

16. Thompson JR, Palmer TM, Moreno S. Bayesian analysis in Stata using WinBUGS. STATA J 2006;6:530-49.
17. Havelaar AH, Haagsma JA, Mangen MJ, et al. Disease burden of foodborne pathogens in the Netherlands, 2009. Int J Food Microbiol 2012;156:231-8.

18. Tam CC, O'Brien SJ, Tompkins DS, et al. Changes in causes of acute gastroenteritis in the United Kingdom over 15 years: microbiologic findings from 2 prospective, population-based studies of infectious intestinal disease. Clin Infect Dis 2012;54:1275-86.

19. Gormley FJ, Little CL, Rawal N, et al. A 17-year review of foodborne outbreaks: describing the continuing decline in England and Wales (1992-2008). Epidemiol Infect 2011;139:688-99.

20. Stephen DM, Barnett AG. Effect of temperature and precipitation on salmonellosis cases in South-East Queensland, Australia: an observational study. BMJ Open 2016;6:e010204.

21. Tariq L, Haagsma J, Havelaar A. Cost of illness and disease burden in The Netherlands due to infections with Shiga toxin-producing Escherichia coli O157. J Food Prot 2011;74: 545-52.

22. Havelaar $\mathrm{AH}$, de Wit MA, van Koningsveld $\mathrm{R}$, et al. Health burden in the Netherlands due to infection with thermophilic Campylobacter spp. Epidemiol Infect 2000;125:505-22. 